\title{
BMJ Open Evaluation of an alternative care provider clinic for severe sleep- disordered breathing: a study protocol for a randomised controlled trial
}

\author{
Ada Ip-Buting, ${ }^{1}$ Jenny Kelly, ${ }^{1}$ Maria J Santana, ${ }^{1,2}$ Erika D Penz, ${ }^{3}$ \\ W Ward Flemons, ${ }^{1,4,5}$ Willis H Tsai, ${ }^{2,4,5}$ Kristin L Fraser, ${ }^{4,5}$ Patrick J Hanly, ${ }^{4,5}$ \\ Sachin R Pendharkar ${ }^{1,2,4,5}$
}

To cite: Ip-Buting A, Kelly J, Santana MJ, et al. Evaluation of an alternative care provider clinic for severe sleepdisordered breathing: a study protocol for a randomised controlled trial. BMJ Open 2017;7:e014012. doi:10.1136/bmjopen-2016014012

- Prepublication history and additional material is available. To view please visit the journal (http://dx.doi.org/ 10.1136/bmjopen-2016014012).

Received 24 August 2016 Revised 21 February 2017 Accepted 6 March 2017

CrossMark

For numbered affiliations see end of article.

Correspondence to Dr Sachin R Pendharkar; Sachin.pendharkar@ucalgary.ca

\section{ABSTRACT}

Introduction: Despite the high prevalence of sleepdisordered breathing (SDB) and the significant health consequences associated with untreated disease, access to diagnosis and treatment remains a challenge. Even patients with severe SDB (severe obstructive sleep apnoea or hypoventilation), who are at particularly high risk of adverse health effects, are subject to long delays. Previous research has demonstrated that, within a sleep clinic, management by alternative care providers (ACPS) is effective for patients with milder forms of SDB. The purpose of this study is to compare an ACP-led clinic (ACP Clinic) for patients with severe SDB to physician-led care, from the perspective of clinical outcomes, health system efficiency and cost.

Methods and analysis: The study is a randomised, controlled, non-inferiority study in which patients who are referred with severe SDB are randomised to management by a sleep physician or by an ACP. ACPS will be supervised by sleep physicians for safety. The primary outcome is positive airway pressure (PAP) adherence after 3 months of therapy. Secondary outcomes include: long-term PAP adherence; clinical response to therapy; health-related quality of life; patient satisfaction; healthcare usage; wait times from referral to treatment initiation and cost-effectiveness. The economic analysis will be performed using the perspective of a publicly funded healthcare system. Ethics and dissemination: Ethics approval was obtained from the Conjoint Health Research Ethics Board (ID: REB13-1280) at the University of Calgary. Results from this study will be disseminated through presentations at scientific conferences and publication in peer-reviewed journals.

Trial registration number: NCT02191085; Pre-results.

\section{INTRODUCTION}

\section{Background and rationale}

Sleep-disordered breathing (SDB) is common and has significant medical consequences. The most common type of SDB, obstructive

\section{Strengths and limitations of this study}

- This is a randomised controlled non-inferiority trial comparing alternative care provider care to traditional physician-led care for patients with severe sleep-disordered breathing.

- The primary outcome of treatment adherence is objective and important for this patient population, who are at greater risk of medical complications.

- The comprehensive evaluation strategy includes clinical and health system outcomes, including its effect on timely access to care and economic impacts.

- This is a single centre study that uses nonphysician healthcare providers who may not be available in other sleep clinics or jurisdictions.

sleep apnoea (OSA) affects up to $24 \%$ of men and $9 \%$ of women. ${ }^{1}$ Untreated severe OSA has been associated with an increased risk of cardiovascular disease, including hypertension, stroke, and fatal and non-fatal cardiovascular events. $^{2-5}$ Additionally, patients with OSA are at increased risk for motor vehicle crashes, ${ }^{6}$ use more healthcare resources, ${ }^{7}$ and may experience reduced survival compared with those without OSA. ${ }^{8}$ The economic impact of OSA in the USA has been estimated at US\$3.5 billion/year, with the mean annual cost of healthcare usage and treatment of medical consequences of OSA exceeding agematched and sex-matched controls twofold to threefold. ${ }^{9}$ Treatment of OSA with continuous positive airway pressure (CPAP) reduces cardiovascular risk, motor vehicle crashes and healthcare usage, and is cost-effective. ${ }^{2}$ 9-12

Hypoventilation, defined as a daytime partial pressure of arterial carbon dioxide $\geq 45 \mathrm{~mm} \mathrm{Hg}$ or an increase in nocturnal transcutaneous carbon dioxide $\geq 10 \mathrm{~mm} \mathrm{Hg}$ occurs in up to 
$20 \%$ of patients with OSA, ${ }^{13}$ and can also occur in obese patients and in association with other common respiratory diseases such as chronic obstructive pulmonary disease. Patients with hypoventilation use more healthcare resources than the general population ${ }^{14}$ and are at an increased risk of acute respiratory failure, hospital admission and death. ${ }^{15}$ Treatment of hypoventilation with positive airway pressure (PAP) therapy, with or without supplemental oxygen, reduces the number of days in hospital and may reduce physician visits. ${ }^{14} 1617$ However, ideal treatment for those with hypoventilation requires overnight monitoring of PAP initiation to determine if CPAP alone is safe and effective, or if additional therapy (non-invasive ventilation (NIV), supplemental $\mathrm{O}_{2}$ ) is required. In contrast, those patients without hypoventilation can be safely treated with home auto-CPAP titration.

The difficulty in providing timely access to sleep specialists is widespread. Delayed access for sleep care have been reported in Canada, ${ }^{18}$ the USA, Europe and Australia. ${ }^{19}$ These delays are particularly important for patients with severe SDB (severe OSA and/or hypoventilation) due to the increased risk of adverse clinical outcomes. Current strategies to improve timely access include the use of home sleep apnoea testing (HSAT) or telemedicine consultation with a sleep physician; ${ }^{20-23}$ these strategies may be particularly beneficial for patients residing in rural areas or those with mobility concerns. However, their success relies on the supply of sleep physicians for consultation, which is inadequate in many jurisdictions. ${ }^{24}$ The use of trained non-physician alternative care providers (ACPs) or primary care physicians to manage patients with SDB has been proposed to improve access to care and to reduce wait times. ACPs, such as nurses and respiratory therapists, have been shown to be effective substitutes for sleep physicians in the management of uncomplicated patients. ${ }^{25}{ }^{26}$ Management by primary care physicians has also been demonstrated to be non-inferior to sleep specialist care. ${ }^{27} 28$

The above studies have identified potential roles for ACPs and primary care physicians in the management of uncomplicated OSA, but no study has examined whether anyone but a sleep physician can manage patients with more severe forms of SDB such as hypoventilation. It is likely, given the complexity of managing patients who may need more advanced PAP therapies and possibly supplemental oxygen, that these patients are best cared for within a sleep clinic and not in the primary care setting. It is unknown whether non-physician providers within a sleep clinic can safely and effectively diagnose and treat complex patients with severe SDB. Furthermore, the impact on wait times or the quality of patient care have not been evaluated.

Given the high risk of adverse health outcomes related to untreated severe SDB, and prompted by wait times that far exceed the current Canadian guideline of 4 weeks from referral to assessment, we designed an ACP Clinic for patients with severe SDB. This paper provides a description of the model and the protocol for its implementation and evaluation. The rationale for publishing this protocol is to highlight the importance of improving access to care for this patient population, to consider an expanded scope of practice and service delivery for ACPs within a sleep clinic, and to describe our comprehensive evaluation of clinical and health system outcomes.

\section{Objectives}

The study is a randomised, controlled, non-inferiority study evaluating the 3-month and 1-year outcomes of an ACP Clinic for patients referred with suspected severe SDB. Patients will be randomised to one of two treatment arms-standard management by sleep physicians or management by ACPs in the ACP Clinic. The study hypotheses are that, compared with a traditional physician-led approach, the ACP Clinic will:

1. result in similar PAP treatment adherence, subjective and objective response to therapy, health-related quality of life (HRQL) and patient satisfaction 3 months and 1 year after treatment initiation;

2. reduce the time from referral to treatment initiation;

3. result in similar number of sleep physician visits or diagnostic tests during the first year of treatment initiation;

4. be cost-effective during the first year of treatment initiation.

\section{METHOD AND ANALYSIS}

\section{Study setting}

The Foothills Medical Centre (FMC) Sleep Centre is a publicly funded, tertiary academic sleep centre in Calgary, Alberta, with a catchment area of $\sim 2$ million people in Alberta and British Columbia. There are eight sleep specialist physicians at the FMC Sleep Centre (six respirologists, one psychiatrist and one neurologist).

The FMC Sleep Centre receives $\sim 2500$ referrals annually, of which $60 \%$ are for SDB and $30 \%$ are for severe SDB. Twenty-five polysomnograms (PSG) and 75 HSAT are performed each week and all tests are interpreted by a sleep physician. All newly referred patients with suspected SDB undergo HSAT prior to the initial assessment by a sleep physician. Consistent with current Canadian guidelines, ${ }^{29}$ patients are prioritised based on severity of SDB on HSAT, medical comorbidity, daytime sleepiness and whether they work in a safety-critical occupation. Patients are assigned to sleep physicians based on the suspected diagnosis. Any physician at the FMC Sleep Centre may assess patients with uncomplicated OSA, but prior to this study, patients with suspected severe SDB were only scheduled to see respirologists. Patients may undergo PSG at the physician's discretion if the diagnosis is uncertain or if there is a concern about ambulatory PAP auto-titration.

Consistent with current clinical guidelines, ${ }^{30}{ }^{31}$ patients with mild or moderate SDB may be offered PAP therapy or referral to a dentist for oral appliance 
therapy, depending on symptoms and patient preference. However, PAP is recommended as first-line therapy for all patients with severe SDB. Patients are also counselled on lifestyle modification (eg, weight loss, avoidance of excessive alcohol and sedative use) as appropriate. Conventional practice at the FMC Sleep Centre is that ACPs conduct follow-up assessments, either in the clinic or by telephone, guided by sleep physician-approved protocols. For patients with uncomplicated OSA, we have previously demonstrated that this is an effective follow-up model. ${ }^{26}$ The primary sleep physician could be reconsulted by the ACP as necessary; physician reassessment could occur by review of the case with the ACP or direct follow-up with the patient.

\section{Alternative care providers}

All ACPs are respiratory therapists (RT) who have completed a 2-year accredited respiratory therapy training programme in Canada. Completion of this programme includes attendance of 300 hours of classroom and laboratory-based learning on respiratory physiology and over 800 hours of education and supervised clinical experience with invasive and non-invasive mechanical ventilation in a variety of medical contexts. ${ }^{32}$ In addition, ACPs at the FMC Sleep Centre have at least 5 years of experience assessing and managing patients with SDB. All ACPs are registered with the provincial respiratory therapy professional college in Alberta, Canada, which regulates RTs based on established standards of practice and continuing education requirements.

The scope of ACP practice at the FMC Sleep Centre is defined by a physician-approved policy that complies with provincial regulations for Registered RTs. ACP activities include: initial education about PAP therapy; follow-up assessment of patients on PAP; and ordering of arterial blood gas and HSAT. ACPs can also make adjustments to PAP equipment, including humidity, ramp and expiratory pressure release settings and small pressure changes to CPAP without a physician prescription. Additionally, regular review of ACP protocols for the management of patients on PAP and education on respiratory and nonrespiratory sleep disorders occur bi-weekly at ACP-focused case conferences.

\section{Eligibility criteria}

Patients who are referred to the FMC Sleep Centre will be eligible to participate in the study if they meet at least one of the following inclusion criteria:

1. Respiratory Disturbance Index (RDI) $\geq 30$ events/ hour on HSAT;

2. mean nocturnal oxygen saturation $\leq 85 \%$ on HSAT;

3. suspected sleep hypoventilation syndrome, defined by an RDI $\geq 15$ events/hour on HSAT and partial pressure of carbon dioxide $\geq 45 \mathrm{~mm} \mathrm{Hg}$ on arterial blood gas while awake.

Patients already on supplemental oxygen, in whom HSAT is insensitive for the diagnosis of OSA, will be recruited if the airflow channel on the HSAT indicates severe SDB or if the investigators determine that the clinical suspicion of severe SDB is high.

Patients will be excluded from the study if they have a suspected concomitant sleep disorder other than SDB such as insomnia or narcolepsy, have previously been treated with PAP therapy for SDB, have primary health insurance from outside Alberta due to difficulties in collecting healthcare usage data, or if they fail to provide consent to participate in the study.

\section{Randomisation and blinding}

Participants will be randomly assigned to either the standard management by a sleep physician or to the ACP Clinic with a 1:1 allocation using a computer generated randomisation schedule.

The allocation sequence will be concealed until the patient is eligible and consented to the study. The research associates will allocate patients according to the randomisation schedule and the booking clerk will schedule the patient with the appropriate provider according to the FMC Sleep Centre scheduling policies. The allocation of the participants will be stored in a database, which is only accessible to the research associate. If the patient prefers to be assessed by a particular provider at the time of initial scheduling, then the allocation would be discontinued and the patient would be excluded from the study.

The research associate and booking clerks will not be blinded to the study. The ACPs and sleep physicians, including investigators, will not be able to identify study patients as both groups will be conducting clinics comprised of a combination of study and non-study patients and there will be no other indications that the patient is a study participant. Owing to the nature of the study, the participants will not be blinded to the study.

\section{Intervention}

Figure 1 presents an overview of study flow. Patients randomised to standard management will receive usual care as described above (see the 'Study setting'). Patients will undergo an initial assessment by a sleep physician, who will establish a management plan with the patient that may include PSG, initiation of therapy for SDB and/or clinical follow-up. Follow-up may be delegated to an ACP, who will manage patients within their scope of practice as defined in existing physician-approved protocols. As is routine practice at the FMC Sleep Centre, ACPs will be able to refer patients back to the primary sleep physician for persistent symptoms of SDB or management of clinical issues outside of their scope of practice.

Patients randomised to care in the ACP Clinic will have an initial assessment by an ACP, during which time the management plan will be established by the ACP and the patient. Since this patient population is medically complex, the management plan will be reviewed with a sleep physician immediately after the assessment. The sleep physician will meet briefly with the patient to 
Figure 1 Patient flow diagram.

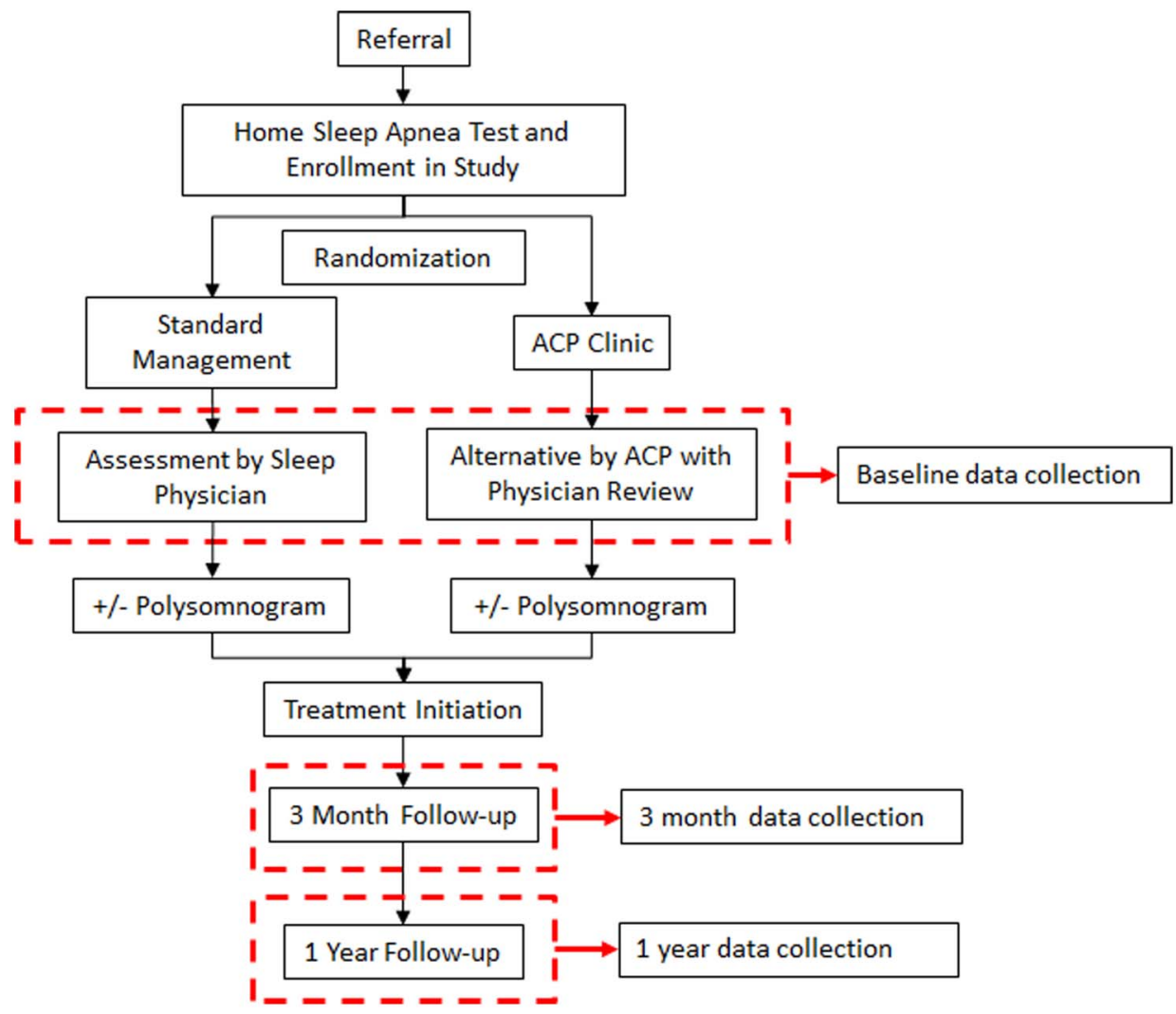

review any additional medical or sleep-related concerns before confirming the proposed plan for further testing and/or treatment. The presence of a sleep physician in the clinic was also deemed important in case a patient was unstable at the time of assessment (eg, severe hypoxemia, decompensated respiratory failure). Follow-up will occur with the ACP to review test results, discuss and initiate treatment and to assess treatment response. The ACP will be able to refer patients back to the primary sleep physician as in the usual care group.

In both groups, as is standard practice at the FMC Sleep Centre, HSAT requisitions will be completed by physicians or ACPs and interpreted by a sleep physician. PSG requisitions and interpretation, and prescriptions for PAP therapy will be completed by the primary sleep physician.

\section{Outcomes}

A summary of study outcomes and when they will be measured is presented in table 1 .

\section{Primary outcome}

The primary outcome is PAP adherence after 3 months of therapy. Treatment adherence will be reported in terms of average nightly use and dichotomised based on whether patients used PAP therapy for at least 4 hours a night for at least $70 \%$ of nights. ${ }^{33}$ Adherence downloads from the preceding 4 weeks will be obtained from each patient's PAP machine.
PAP adherence was chosen as a primary outcome for several reasons. First, many outcome measures such as daytime sleepiness, quality of life or functional status are related to PAP use. ${ }^{33-35}$ In addition, many of the outcome measures used in other studies are subjective; patients often underestimate their symptoms leading to relative insensitivity of symptom scores as measures of treatment effectiveness. $^{36-38}$

Second, treatment of severe SDB with PAP is associated with reductions in the risk of cardiovascular disease, development of metabolic disorders such as dia-

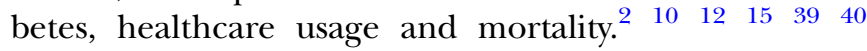
These are important clinical outcomes for individual patients $^{34}$ and for health system usage and cost. Given that the population under study is at particularly high risk for these adverse medical consequences, we determined that treatment adherence was of a higher priority than other outcomes for a comprehensive evaluation of this novel model of care for severe SDB.

Finally, PAP adherence is commonly used as an outcome measure in studies examining service delivery models for patients with $\mathrm{SDB},{ }^{20} 28^{41-45}$ and has been identified as an indicator of high-quality care for SDB. ${ }^{46}$

\section{Secondary outcomes}

A number of secondary outcomes, related to clinical effectiveness, healthcare usage, system efficiency and cost, will be analysed. Additional details on the secondary outcomes are available in the online supplementary material. The secondary outcomes include: 
Table 1 Outcome measures collection points

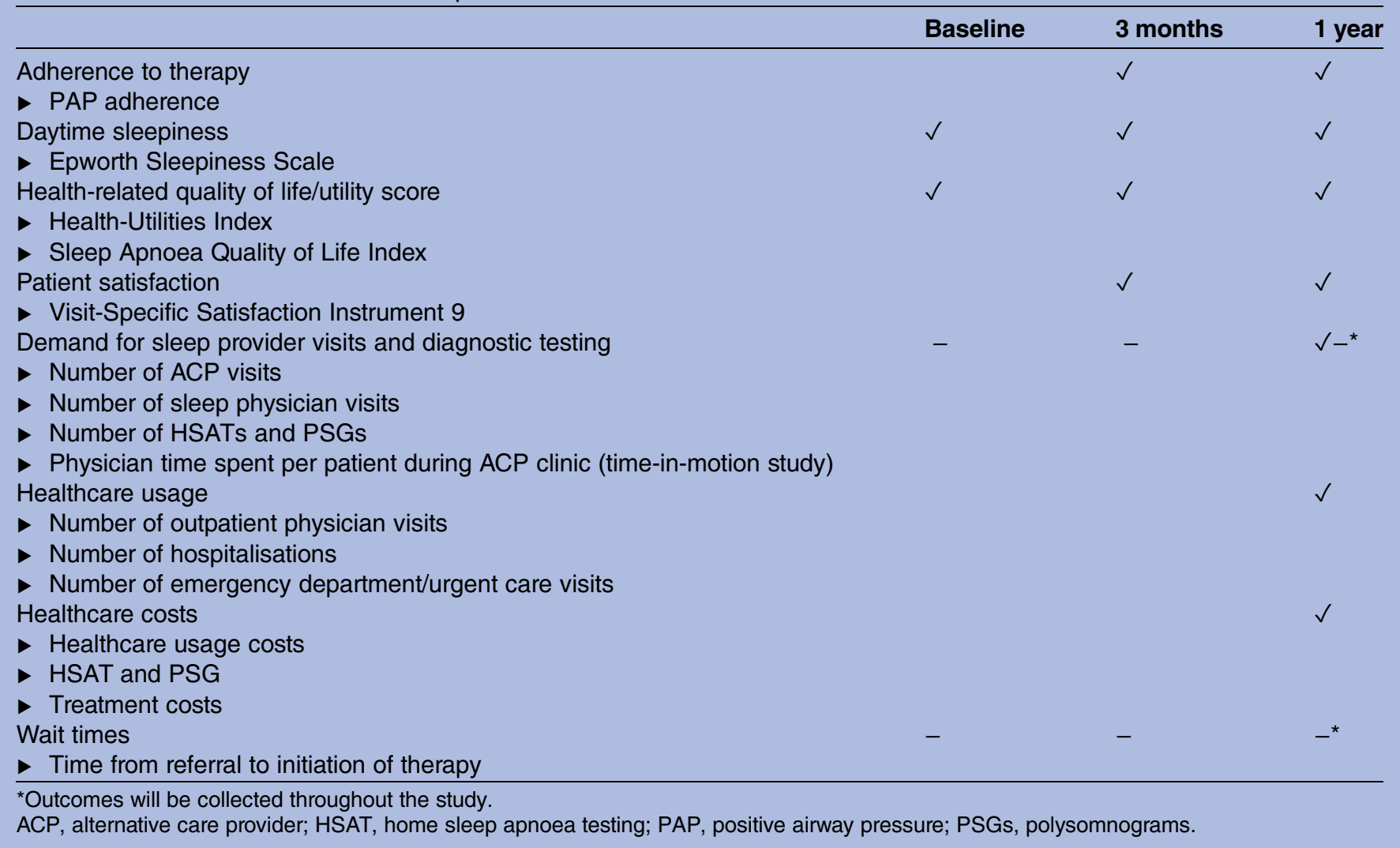

\section{Long-term PAP adherence}

PAP adherence will be measured after 1 year of therapy.

\section{Daytime sleepiness}

The Epworth Sleepiness Scale is a validated patient questionnaire assessing daytime sleepiness.

\section{Health-related quality of life (HRQL)}

HRQL will be measured using general and diseasespecific instruments. The Health-Utilities Index (HUI) and the short-form Sleep Apnoea Quality of Life Index (SAQLI) will be used to measure general HRQL and disease-specific HRQL, respectively.

\section{Patient satisfaction}

The Visit-Specific Satisfaction Instrument (VSQ-9) is a validated measure of patient satisfaction with an outpatient visit. $^{47}$

\section{Demand for sleep provider visits and diagnostic sleep testing}

Data on demand will include patient visits to physicians and ACPs, and all HSAT and PSG testing. We will also capture written communication between physicians and ACPs regarding the management of study patients.

\section{Healthcare usage}

Data on healthcare use will include outpatient physician visits, hospitalisations and emergency department or urgent care visits from referral to 1 year following treatment initiation.

\section{Cost effectiveness}

Costs captured from referral to 1 year after treatment initiation will be summarised in the following categories: sleep physician and ACP visits; sleep investigations; treatment costs and healthcare usage costs. Cost-effectiveness will be measured from the perspective of a publicly funded healthcare system.

\section{Wait times}

Time to treatment initiation will be evaluated for patients in each arm. Although wait times for initial assessment may be shorter for the ACP Clinic based on the addition of ACP supply, time to treatment initiation incorporates delays related to PSG and additional physician or ACP visits before a treatment decision is made. Time to treatment initiation is also a clinically relevant outcome, particularly for patients with severe SDB.

\section{Statistical analysis}

This study will use a modified intention to treat analysis, in which results will be analysed for patients who are randomised and have treatment adherence data 3 months after initiating PAP therapy.

Paired t-tests will be used to compare clinical outcomes from baseline to 3 months and 1 year and unpaired t-tests will be used to compare time to 
treatment initiation and measures of patient demand. Multiple logistic regression will be used to identify predictors of study outcomes using variables identified as predictive on univariate regression. Outcomes will be transformed into binary variables based on clinically relevant cut-offs.

The economic analysis will be performed using the perspective of a publicly funded healthcare system. An estimate of mean utility scores based on the HUI questionnaire will be calculated and the average quality-adjusted life-year (QALY) will be calculated for each study arm. Using bootstrapping, we will compare the difference in mean costs and QALYs between the study arms to generate an incremental costeffectiveness ratio for an ACP-led clinic compared with traditional physician-led care. Sensitivity analyses will be performed to evaluate the effect of changes in physician fees, RT salaries, diagnostic testing costs and treatment costs.

All study outcomes will be analysed in different subgroups to clarify the impact of ACP-led care for patients with different patient flow pathways or clinical treatments. Prespecified subgroups include:

1. patients who undergo PSG versus patients who do not undergo PSG;

2. patients treated with bi-level PAP versus patients treated with CPAP;

3. patients who are treated with oxygen versus patients treated without oxygen.

\section{Sample size}

The study is powered to assess the non-inferiority of ACP-led care compared with usual care by sleep physicians. A non-inferiority margin of -1 hour of PAP adherence was determined by consensus of the investigators and has been used in previous studies comparing different models of care for SDB. ${ }^{28}$ The study will require 138 patients (69 in each arm) to achieve $90 \%$ power with a type I error of 0.05 , using this non-inferiority margin and a SD of 2 hours of nightly CPAP use. ${ }^{48}$ The SD used in the sample size calculation was based on the results of two previous studies. ${ }^{33}{ }^{34}$ To account for withdrawals and loss to follow-up $(\sim 15 \%$ at the FMC Sleep Centre), recruitment will continue until 3-month adherence data is available for 150 patients.

\section{Trial status}

Patient recruitment began in October 2014 and was completed in August 2016. We are in the process of collecting baseline measurements, 3-month and 1-year follow-up measurements and anticipate the completion of 3-month and 1-year data collection by December 2016 and December 2017, respectively.

\section{ETHICS AND DISSEMINATION}

The ethics approval process involved reviewing the study with respect to content and compliance with applicable research and safety regulations. In addition to the initial approval of the study, the CHREB will review the study on an annual basis. Any modification to the study protocol will require a formal amendment to the protocol and submitted to the CHREB for approval.

In addition, the study is registered under Clinicaltrials. gov (ID: NCT02191085). Changes or updates to the study, including the study protocol, must be made at least every 12 months. Prior to the completion of the study, the record must be reviewed every 6 months.

The principal investigator and the attending physician are responsible for assessing, reporting and managing solicited and spontaneously reported adverse events and other unintended effects of trial interventions or trial conduct.

\section{Data quality and management}

To promote data quality, the research associates will randomly select $10 \%$ of eligible patients and review the data entered and collected. Range check for data values will be performed for the entire data set.

In accordance with research ethics board approval, all data will be stored on a secure network drive within University of Calgary firewalls and will be accessed by the principal investigator and research associate using password-protected study computers through a Virtual Private Network. Identifying information will be replaced with a unique identifier in any data that is reviewed by investigators, with the associated identifying data stored in a separate password-protected file and accessible only to the research associate and principal investigator for the purposes of reconciling data errors. The clinical members of the research team may have access to clinical information on study patients as part of clinical care, but will not have access to identifiable patient records within the research database. The final data set, without any identifiable information, will be accessible to the principal investigator. The research team may access the data set on request.

\section{Consent and withdrawal}

Patients will be recruited by a research associate who is not involved in the clinical management of SDB. Written informed consent will be obtained from patients prior to any participation in the study. The research associates will contact patients prior to the 3-month and 1-year follow-up appointments to promote participant retention and complete follow-up.

Participants can withdraw from the study at any time without any consequences. At time of withdrawal, the participant will be asked if additional healthcare usage data can be collected. If the participant chooses not to participate in further data collection, data contributed up to the point of withdrawal will be retained but no further data will be collected.

\section{Dissemination}

Results will be disseminated through publications in peer-reviewed journals; one manuscript will report the 
3-month clinical data, and a second manuscript will include longer term clinical and health system outcomes after 1 year. Publications from this research will add to the emerging literature on novel models of care for SDB, and in particular will address whether ACPs can be used to manage a more severe subset of patients. This study lays the foundation for additional research to explore the optimal way to deliver care in the context of such a highly prevalent disease.

The results of this study will also be shared with operational leaders at the FMC Sleep Centre, to determine whether the ACP Clinic model is effective for patients. We will also disseminate the protocol and the study findings to other centres within and outside of Canada through the Canadian Sleep and Circadian Network, and provide insights on our experience to those groups looking to implement such a programme.

\section{DISCUSSION}

In many jurisdictions, providing timely access to care for patients with SDB is a challenge due to a shortage of sleep physicians. Building on the results of previous studies of alternative models of care delivery for OSA, this study aims to determine whether ACPs can manage patients with severe SDB. If the study aims are met, the role of ACPs in the management of severe SDB will be more clearly understood in terms of their impact on clinical, economic and health system outcomes. The results of this study will help sleep clinicians and health system administrators to determine the optimal scope of practice of ACPs.

As is the case in many healthcare systems with limited access, sleep clinics may adopt strategies that prioritise patients with severe disease above those with milder disease. This strategy allows patients who are at higher risk of medical complications to be assessed sooner and is particularly important when wait times are long. It could be argued that ACPs are best used to manage milder patients as has been demonstrated in previous studies, thus creating capacity for sleep physicians to assess severe patients. However, given the burden of SDB in the population, a significant proportion of whom have severe disease, and insufficient supply of sleep physicians in many jurisdictions, it is probable that delays for severe patients are also long. Furthermore, if ACP capacity could be increased, it is conceivable that wait times for less severe patients might paradoxically be shorter than for more severe patients. Thus, a strategy that aims to directly improve access for higher risk patients, such as using ACPs to manage patients with severe SDB, is preferable to one in which ACPs only assess patients who are of lower priority.

Many healthcare systems have insufficient resources to address this imbalance of supply and demand. This study proposes to mitigate this problem through the use of non-physician healthcare providers, under the hypothesis that ACPs can improve access and are cost- effective. However, when proposing a novel model of care, it is essential to demonstrate clinical effectiveness in addition to evaluating the impacts on the healthcare system. This study will comprehensively evaluate an ACP-led pathway for the management severe SDB, including clinical outcomes as well as demand for healthcare resources and costs.

\section{Limitations}

The proposed study has several limitations. First, it is possible that patient preference for care by a sleep physician may influence study outcomes. Our clinical experience with ACP care at the FMC Sleep Centre has been that patients do not object to this management pathway. Furthermore, a previous study of nurse-led care for OSA demonstrated that patient satisfaction did not suffer with non-physician management and in fact was higher with respect to certain aspects of the healthcare encounter. ${ }^{25}$ The research associate will reassure patients that ACP care will be supervised by a sleep physician and that referral back to a sleep physician can be initiated at any point at the patient's request. Finally, we have selected objective outcomes such as PAP adherence, wait times and healthcare costs to minimise potential confounding by patient perceptions of the care they receive.

A second potential limitation relates to the use of multiple instruments to collect patient reported outcomes. It is possible that patients will experience significant burden from these questionnaires, leading to incomplete or inaccurate data. However, previous work has demonstrated that 'questionnaire fatigue' is not significant in an outpatient setting. ${ }^{49}{ }^{50}$ Additionally, we have performed a small time trial for our battery of instruments with patient engagement researchers and observed that the entire set of questionnaires takes $\sim 15$ min to complete. Consequently, we do not anticipate that the completion of questionnaires will lead to any delays on the day of the visit. Our expectation of no significant adverse impact on clinic flow is consistent with previous literature. ${ }^{50}$

Third, this study uses RTs as alternative care providers. RTs are highly trained in respiratory care and ventilation but are not a recognised health profession in some parts of the world. Thus, the study results might not be generalisable to jurisdictions in which the RT role does not exist. We recognise that an ACP in any clinical setting should have expertise in managing the patient population; in this regard, only RTs with additional expertise in the management of SDB are employed at the FMC Sleep Centre. Similarly, nurses and other healthcare professions should be comfortable with respiratory care and ventilation, and may require specific additional training to achieve this.

Finally, patients who are randomised to the ACP Clinic arm could experience an adverse outcome related to ACP care, such as the need for an emergency department visit or hospitalisation due to unrecognised illness at the time of assessment. This important patient safety 
risk will be mitigated by the assignment of each ACP Clinic patient to a primary sleep physician, who will review the management plan with the ACP and meet each patient at the time of the clinic visit. Furthermore, our experience with the delegation of follow-up care to ACPs at the FMC Sleep Centre suggests that trained RTs appropriately identify complex patients, and obtain guidance either through direct communication or by scheduling a follow-up clinic visit with the sleep physician. While we have mandated physician supervision during the ACP Clinic, we recognise that in other jurisdictions, advanced practice nurses or nurse practitioners may have the expertise to manage these patients independently.

\section{Author affiliations}

${ }^{1}$ W21C Research and Innovation Centre, Cumming School of Medicine, University of Calgary, Calgary, Alberta, Canada

${ }^{2}$ Department of Community Health Sciences, Cumming School of Medicine, University of Calgary, Calgary, Alberta, Canada

${ }^{3}$ College of Medicine, University of Saskatchewan, Saskatoon, Saskatchewan, Canada

${ }^{4}$ Department of Medicine, Cumming School of Medicine, University of Calgary, Calgary, Alberta, Canada

${ }^{5}$ Foothills Medical Centre Sleep Centre, University of Calgary, Calgary, Alberta, Canada

Contributors MJS, EDP, WWF, WHT, KLF, PJH contributed to study conception/design. EDP, WHT, SRP provided statistical expertise, sample size calculation and will lead the analysis. Al-B and JK contributed to data acquisition and drafted this protocol manuscript. All authors revised the paper and approved the final version.

Funding This work was supported by The Lung Association-Alberta \& NWT and by the Canadian Sleep and Circadian Network (funded by the Canadian Institutes of Health Research, grant number 339739-CDP)

Disclaimer The funding source had no role or input in the design of this study and will not have any role or input during its execution, analyses and interpretation of the data, or in the dissemination of the findings.

Competing interests None declared.

Ethics approval Ethics approval was obtained for this study from the Conjoint Health Research Ethics Board (CHREB) at the University of Calgary (Ethics ID: REB13-1280).

Provenance and peer review Not commissioned; externally peer reviewed.

Data sharing statement Study data is not yet available.

Open Access This is an Open Access article distributed in accordance with the Creative Commons Attribution Non Commercial (CC BY-NC 4.0) license, which permits others to distribute, remix, adapt, build upon this work noncommercially, and license their derivative works on different terms, provided the original work is properly cited and the use is non-commercial. See: http:// creativecommons.org/licenses/by-nc/4.0/

\section{REFERENCES}

1. Young T, Palta M, Dempsey J, et al. The occurrence of sleep-disordered breathing among middle-aged adults. N Engl J Med 1993;328:1230-5.

2. Marin JM, Carrizo SJ, Vicente E, et al. Long-term cardiovascular outcomes in men with obstructive sleep apnoea-hypopnoea with or without treatment with continuous positive airway pressure: an observational study. Lancet 2005;365:1046-53.

3. Peppard PE, Young T, Palta M, et al. Prospective study of the association between sleep-disordered breathing and hypertension. N Engl J Med 2000;342:1378-84.
4. Redline S, Yenokyan G, Gottlieb DJ, et al. Obstructive sleep apneahypopnea and incident stroke: the sleep heart health study. Am $J$ Respir Crit Care Med 2010;182:269-77.

5. Yaggi HK, Concato J, Kernan WN, et al. Obstructive sleep apnea as a risk factor for stroke and death. $N$ Engl J Med 2005;353:2034-41

6. George C, Nickerson P, Hanly P, et al. Sleep apnoea patients have more automobile accidents. Lancet 1987;330:447.

7. Ronald J, Delaive K, Roos L, et al. Health care utilisation in the 10 years prior to diagnosis in obstructive sleep apnea syndrome patients. Sleep 1999;22:225-9.

8. Punjabi NM, Caffo BS, Goodwin JL, et al. Sleep-disordered breathing and mortality: a prospective cohort study. PLoS Med 2009;6:e1000132.

9. Tarasiuk A, Reuveni $\mathrm{H}$. The economic impact of obstructive sleep apnea. Curr Opin Pulm Med 2013;19:639-44.

10. Bahammam A, Delaive $\mathrm{K}$, Ronald J, et al. Health care utilisation in males with obstructive sleep apnea syndrome two years after diagnosis and treatment. Sleep 1999;22:740-7.

11. George CF. Reduction in motor vehicle collisions following treatment of sleep apnoea with nasal CPAP. Thorax 2001;56:508-12.

12. Martínez-García MÁ, Galiano-Blancart R, Román-Sánchez $P$, et al. Continuous positive airway pressure treatment in sleep apnea prevents new vascular events after ischemic stroke. CHEST 2005;128:2123-9.

13. Kaw R, Hernandez AV, Walker E, et al. Determinants of hypercapnia in obese patients with obstructive sleep apnea: a systematic review and metaanalysis of cohort studies. CHEST 2009;136:787-96.

14. Berg G, Delaive K, Manfreda J, et al. The use of health-care resources in obesity-hypoventilation syndrome. CHEST 2001;120:377-83.

15. Priou P, Hamel J-F, Person C, et al. Long-term outcome of noninvasive positive pressure ventilation for obesity hypoventilation syndrome. CHEST 2010;138:84-90.

16. Povitz M, Bolo CE, Heitman SJ, et al. Effect of treatment of obstructive sleep apnea on depressive symptoms: systematic review and meta-analysis. PLoS Med 2014;11:e1001762.

17. Povitz M, Hanly PJ, Pendharkar SR, et al. Treatment of sleep disordered breathing liberates obese hypoxemic patients from oxygen. PLOS ONE 2015;10:e0140135.

18. Rotenberg BW, George CF, Sullivan KM, et al. Wait times for sleep apnea care in Ontario: a multidisciplinary assessment. Can Respir J 2010;17:170.

19. Flemons WW, Douglas NJ, Kuna ST, et al. Access to diagnosis and treatment of patients with suspected sleep apnea. Am J Respir Crit Care Med 2004;169:668-72.

20. Kuna ST, Gurubhagavatula I, Maislin G, et al. Noninferiority of functional outcome in ambulatory management of obstructive sleep apnea. Am J Respir Crit Care Med 2011;183:1238-44.

21. Hirshkowitz M, Sharafkhaneh A. A telemedicine program for diagnosis and management of sleep-disordered breathing: the fast-track for sleep apnea tele-sleep program. Semin Respir Crit Care Med 2014;35:560-70.

22. Witmans MB, Dick B, Good J, et al. Delivery of pediatric sleep services via telehealth: the Alberta experience and lessons learned. Behav Sleep Med 2008;6:207-19.

23. Mulgrew AT, Fox N, Ayas NT, et al. Diagnosis and initia management of obstructive sleep apnea without polysomnography: a randomized validation study. Ann Intern Med 2007;146:157-66.

24. Pendharkar SR, Bischak DP, Rogers $P$, et al. Using patient flow simulation to improve access at a multidisciplinary sleep centre. $J$ Sleep Res 2015;24:320-7.

25. Antic NA, Buchan C, Esterman A, et al. A randomized controlled tria of nurse-led care for symptomatic moderate-severe obstructive sleep apnea. Am J Respir Crit Care Med 2009;179:501-8.

26. Pendharkar SR, Dechant A, Bischak DP, et al. An observational study of the effectiveness of alternative care providers in the management of obstructive sleep apnoea. J Sleep Res 2016;25:234-40.

27. Chai-Coetzer CL, Luo YM, Antic NA, et al. Predictors of long-term adherence to continuous positive airway pressure therapy in patients with obstructive sleep apnea and cardiovascular disease in the SAVE study. Sleep 2013;36:1929-37.

28. Sánchez-de-la-Torre M, Nadal N, Cortijo A, et al. Role of primary care in the follow-up of patients with obstructive sleep apnoea undergoing CPAP treatment: a randomised controlled trial. Thorax 2015;70:346-52.

29. Fleetham J, Ayas N, Bradley D, et al. Canadian Thoracic Society 2011 guideline update: diagnosis and treatment of sleep disordered breathing. Can Respir J 2011;18:25. 
30. Fleetham J, Ayas N, Bradley D, et al. Canadian Thoracic Society guidelines: diagnosis and treatment of sleep disordered breathing in adults. Can Respir J 2006;13:387.

31. Epstein LJ, Kristo D, Strollo PJ Jr, et al Clinical guideline for the evaluation, management and long-term care of obstructive sleep apnea in adults. J Clin Sleep Med 2009;5:263-76.

32. CSRT position statement: the respiratory therapist's role in mechanical ventilation: Canadian Society of Respiratory Therapists, 2013.

33. Kribbs NB, Pack Al, Kline LR, et al. Objective measurement of patterns of nasal CPAP use by patients with obstructive sleep apnea. Am Rev Respir Dis 1993;147:887-95.

34. Weaver TE, Maislin G, Dinges DF, et al. Relationship between hours of CPAP use and achieving normal levels of sleepiness and daily functioning. Sleep 2007;30:711.

35. Antic NA, Catcheside P, Buchan C, et al. The Effect of CPAP in normalizing daytime sleepiness, quality of life, and neurocognitive function in patients with moderate to severe OSA. Sleep 2011;34:111-19.

36. Ahmed I, Thorpy MJ. Determinants and measures of daytime sleepiness. In: Pagel FJ, Pandi-Perumal RS, eds. Primary care sleep medicine: a practical guide. New York, NY: Springer New York, 2014:99-106.

37. Leclerc G, Lacasse Y, Page D, et al. Do obstructive sleep apnea syndrome patients underestimate their daytime symptoms before continuous positive airway pressure treatment?. Can Respir J 2014:21:216-20.

38. Guimarães C, Martins MV, Vaz Rodrigues L, et al. Epworth Sleepiness Scale in obstructive sleep apnea syndrome-an underestimated subjective scale. Rev Port Pneumol 2012;18:267-71.

39. Campos-Rodriguez F, Pena-Grinan N, Reyes-Nunez N, et al. Mortality in obstructive sleep apnea-hypopnea patients treated with positive airway pressure. CHEST 2005;128:624-33

40. Marin JM, Soriano JB, Carrizo SJ, et al. Outcomes in patients with chronic obstructive pulmonary disease and obstructive sleep apnea: the overlap syndrome. Am J Respir Crit Care Med 2010;182:325-31.
41. Bouloukaki I, Giannadaki K, Mermigkis C, et al. Intensive versus standard follow-up to improve continuous positive airway pressure compliance. Eur Respir J 2014;44:1262-74.

42. Pamidi S, Knutson KL, Ghods F, et al. The impact of sleep consultation prior to a diagnostic polysomnogram on continuous positive airway pressure adherence. CHEST 2012;141:51-7.

43. Rosen CL, Auckley D, Benca R, et al. A multisite randomized trial of portable sleep studies and positive airway pressure autotitration versus laboratory-based polysomnography for the diagnosis and treatment of obstructive sleep apnea: the HomePAP study. Sleep 2012;35:757-67.

44. Hoy CJ, Vennelle M, Kingshott RN, et al. Can intensive support improve continuous positive airway pressure use in patients with the sleep apnea/hypopnea syndrome?. Am J Respir Crit Care Med 1999;159:1096-100.

45. Parthasarathy S, Subramanian S, Quan SF. A multicenter prospective comparative effectiveness study of the effect of physician certification and center accreditation on patient-centered outcomes in obstructive sleep apnea. J Clin Sleep Med 2014;10:1-7.

46. Aurora RN, Collop NA, Jacobowitz O, et al. Quality measures for the care of adult patients with obstructive sleep apnea. J Clin Sleep Med 2015;11:357-83.

47. Ware JE, Hays RD. Methods for measuring patient satisfaction with specific medical encounters. Med Care 1988;26:393-402.

48. Julious SA. Sample sizes for clinical trials with normal data. Stat Med 2004;23:1921-86.

49. Santana M-J, Feeny DH. Using the health utilities index in routine clinical care: process, feasibility, and acceptability: a randomized controlled trial. Patient 2009;2:159-67.

50. Santana MJ, Feeny D, Weinkauf $\mathrm{J}$, et al. The use of patient-reported outcomes becomes standard practice in the routine clinical care of lung-heart transplant patients. Patient Relat Outcome Meas 2010;1:93-105 